Article

\title{
Tone Entropy Analysis of Foetal Heart Rate Variability
}

\section{Ahsan Khandoker ${ }^{1,2, *}$, Chandan Karmakar ${ }^{1,5}$, Yoshitaka Kimura ${ }^{3,4}$, Miyuki Endo ${ }^{3}$, Sayaka Oshio ${ }^{3}$ and Marimuthu Palaniswami ${ }^{1}$}

1 Biomedical Engineering Department, Khalifa University of Science, Technology and Research, Abu Dhabi 127788, United Arab Emirates

2 Department of Electrical \& Electronic Engineering, University of Melbourne, Melbourne, VIC 3010, Australia; E-Mails: karmakar@unimelb.edu.au (C.K.); palani@unimelb.edu.au (M.P.)

3 Institute of International Advanced Interdisciplinary Research, Tohoku University School of Medicine, Sendai 980-8577 Japan; E-Mails: ykimura@med.tohoku.ac.jp (Y.K.); miyukien@med.tohoku.ac.jp (M.E.); sayakannbou@hotmail.com (S.O.)

4 Department of Gynecology and Obstetrics, Tohoku University Hospital, Sendai 980-8577, Japan

5 Centre for Pattern Recognition and Data Analytics, Deakin University, VIC 3216, Australia

* Author to whom correspondence should be addressed; E-Mail: ahsank@ieee.org; Tel.: +971-(0)2-501-8559; Fax: +971-(0)2-447-2442.

Academic Editor: Niels Wessel

Received: 17 November 2014 / Accepted: 25 February 2015 / Published: 2 March 2015

\begin{abstract}
Development of the foetal autonomic nervous system can be indirectly understood by looking at the changes in beat to beat variability in foetal heart rates. This study presents Tone-Entropy (T-E) analysis of foetal heart rate variability (HRV) at multiple lags (1-8) to understand the influence of gestational ages (early and late) on the development of the foetal autonomic nervous system (ANS). The analysis was based on foetal electrocardiograms (FECGs) of 46 healthy foetuses of 20-32 weeks (early group) and 22 foetuses of 35-41 weeks (late group). Tone represents sympatho-vagal balance and entropy the total autonomic activities. Results show that tone increases and entropy decreases at all lags for the late foetus group. On the other hand, tone decreases and entropy increases at lags 1-4 in the early foetus group. Increasing tone in late foetuses might represent significant maturation of sympathetic nervous systems because foetuses approaching to delivery period need increased sympathetic activity. T-E could be quantitative clinical index to determine the early foetuses from late ones on the basis of maturation of autonomic nervous system.
\end{abstract}


Keywords: foetal electrocardiogram; foetal heart rate variability; Tone-Entropy analysis; foetal autonomic nervous system

\section{Introduction}

Foetal well-being during pregnancy has been widely evaluated with fluctuations of foetal heart rate or foetal heart rate variability (fHRV) monitoring. However its usefulness in detecting foetal acidemia has recently been questioned [1]. One reason is that clinical interpretation can sometimes differ because beat to beat fetal heart rates were not extracted from foetal electrocardiogram signals which is the most reliable method for quantifying beat to beat heart rates. Early in gestation the foetal heart rate is predominately under the control of the sympathetic nervous system and arterial chemoreceptors [2]. As the foetus develops its heart rate decreases in response to parasympathetic (vagal stimulation) nervous system maturation and variability becomes more pronounced [3]. In the normal foetus, there is an interplay between the sympathetic (acceleration) and parasympathetic (deceleration) nervous systems in the control of heart rate. These systems exert their control via the cerebral cortex, the medulla oblongata, the sympathetic ganglia and the vagus nerve. The interaction between these systems results in a difference in the beat-to-beat intervals resulting in variability of the foetal heart rate tracing. In the term foetus, moderate variability is considered normal as it indicates a normally functioning central nervous system. Beat-to-beat or short-term variability is the oscillation of the FHR around the baseline in amplitude of 5 to $10 \mathrm{bpm}$. Long-term variability is a somewhat slower oscillation in heart rate and has a frequency of three to 10 cycles per minute and an amplitude of 10 to $25 \mathrm{bpm}$. Clinically, loss of beat-to-beat variability is more significant than loss of long-term variability [4]. Gestational age is an important factors that require consideration when assessing the foetal heart rate variability. The completion of the 35 gestational week marks a developmental milestone that is associated with a dramatically decreasing risk for the neonate in the case of a preterm delivery. About $90 \%$ of babies born this week can survive. Lungs are almost fully developed.

A previous study has shown that sympatho-vagal balance in adults can be detected even in the time domain through the Tone-Entropy (T-E) [5]. Tone was verified to reflect the sympatho-vagal balance by a pharmacological experiment where Tone changed in value consistently in a heart rate recovery experiment after exercise where the parasympathetic division became predominant [5]. The T-E evaluation process is not influenced by the time period of data acquisition, nor the baseline heart rate. In addition, the T-E data processing has no signal deformation process such as filtering, window, or limiting process. A very important advantage is that there is no need to control respiration rate in the T-E method, allowing data to be obtained in a natural process [6]. We have recently reported that multi-lag T-E analysis can overcome the limitations of the present practice of single lag T-E analysis in HRV studies [7] because a heartbeat influences not only the beat immediately following it, but also up to $6-10$ beats downstream [8].

Therefore, the aim of this study was to elucidate progressive development of cardiac autonomic functions in healthy foetuses before and after 35 weeks of gestation by multi-lag T-E analysis of foetal heart hearts extracted from foetal electrocardiograms. 


\section{Methods}

\subsection{Data}

Recording of the abdominal ECG signals from 68 pregnant women at the gestational age of 20-41 weeks with normal single pregnancies were collected from Tohoku University Hospital. Out of them 46 cases were before 35 weeks $(G 1 \in[20,32])$ and 22 were after 35 weeks $(G 2 \in[35,41])$ of gestational age. All recordings (each of 1 minute's length) were sampled at $1000 \mathrm{~Hz}$ with 16-bit resolution. The study protocol was approved by Tohoku University Institutional Review Board and written informed consent was obtained from all subjects.

FECG traces were extracted using a method that combines cancellation of the mother's ECG signal and the blind source separation with reference (BSSR) as described in our earlier study [9]. Intervals between successive $\mathrm{R}$ waves of the QRS complex (i.e., R-R intervals in seconds) were calculated using the algorithm developed by Pan and Tompkins [10]. Once RR intervals were calculated, the ectopic beat was selected automatically based on a predefined heart rate range. For any RR interval, if the instantaneous heart rate is lesser than 100 beats/min or higher than 240 beats/min then the interval was marked as ectopic and removed from the RR interval time-series. After artifact removal the average length of RR interval time-series for 68 recordings was 142 beats.

\subsection{Multi-Lag T-E Analysis of HRV Signal}

A RR interval or period is defined as the time difference between two consecutive $R$ peaks of the electrocardiogram (ECG) signal. Let the RR intervals time series $\mathbf{R R}$ be defined as:

$$
R R=\left\{R R_{1}, R R_{2}, R R_{3}, \ldots \ldots, R R_{N}\right\}
$$

where, $N$ is the number RR intervals. Heart rate acceleration and deceleration can be determined from the difference of consecutive $R R$ intervals. If $R R_{i+1}$ become shorter than $R R_{i}$ then it is an acceleration of heart rate. Therefore, acceleration of the heart is expressed as a positive difference and inhibition as a negative difference of RR intervals. However, to reduce the impact of heart rate variation over a wide range of time and different subjects, normalized variation in RR interval is preferred to monitor the variability. In conventional T-E analysis, percentile change of the successive RR intervals with respect to the previous RR interval is expressed as the percentage index $(P I)$ and defined as:

$$
P I_{i}=\frac{R R_{i}-R R_{i+1}}{R R_{i}} \times 100
$$

Tone is defined as a first order moment (arithmetic average) of this PI time series as:

$$
\text { Tone }=\frac{1}{N-1} \sum_{i=1}^{N-1} P I_{i}
$$

Tone is the balance between accelerations (PI $>0)$ and inhibitions $(\mathrm{PI}<0)$ of the heart rate and represents the sympatho-vagal balance faithfully as shown in previous studies [5,11]. Entropy is defined from the probability distribution of PI by using Shannon's formula [12]: 


$$
\text { Entropy }=-\sum_{i=1}^{n} p(i) \log _{2} p(i)
$$

where, $p(i)$ is a probability of $\mathbf{P I}$ having values in the range $i<\mathbf{P I}<i+1$, where $i$ is an integer and $n$ is the number of bin where, $p(i) \neq 0$. The entropy evaluates total acceleration-inhibition activities, or total heart period variations, in a familiar unit of bit.

For multi-lag T-E analysis, we have introduced the $\operatorname{lag}(\mathrm{m})$ in Equation (1), used to derive the PI time series from the RR time series signal. Hence, in the multi-lag T-E analysis PI is expressed as the percent change of the $i$-th and $(i+m)$-th RR intervals with respect to the $i$-th $\mathrm{RR}$ interval and is defined as:

$$
P I_{i}=\frac{R R_{i}-R R_{i+m}}{R R_{i}} \times 100
$$

where, $m$ is an integer and $m=1$ represents the conventional T-E analysis. The detailed methodology of conventional and multi-lag T-E analysis has been described in previous reports $[5,11]$. An important benefit of conventional $(\operatorname{lag}-1) \mathrm{T}$-E evaluation is that it is not influenced by the time period of data acquisition and increasing lag time reintroduces the sensitivity to recording length. We analysed the variation of tone and entropy values with a range of lags $(1 \leq m \leq 8)$.

We quantified several time domain HRV parameters: mean RR, standard deviation of normal RR data $(S D R R)$ and the square root of the mean squared difference of the successive RR data (RMSSD). Spearman correlation analysis was performed to investigate the relationship between T-E values and gestational ages.

\subsection{Statistics}

In this study we have used nonparametric Mann-Whitney U-test for testing the hypothesis regarding median i.e., the median of tone values of $\mathrm{G} 1$ and $\mathrm{G} 2$ are equal. The same test has been used to test the hypothesis for entropy values. Mann-Whitney $U$ test was used since the distribution of features (Tone and entropy) was not normal. A $p$ value of $<0.05$ was considered significant. MATLAB Statistics toolbox was used to perform all statistical operations.

\section{Results}

Figure 1 illustrates typical examples of heart periods (RR intervals), PI time series (\%) and their histograms of foetuses selected from each of the two groups G1 and G2. Alteration of the distributions is clearly discernible. Figure 2 illustrates individual and averaged data of tone and entropy in G1 and G2 groups. A clear tendency was noticed corresponding with the progression of foetal ANS development. Tone increased significantly ( $p<0.05$ ) with late foetuses group (see Table 1 for values) for all lags $(1-8)$, whereas entropy decreased significantly $(p<0.05)$ with the same group for lags up to 4 . Averaged data of the two groups are shown by rectangles (mean \pm SD) and both rectangles were shifted. 

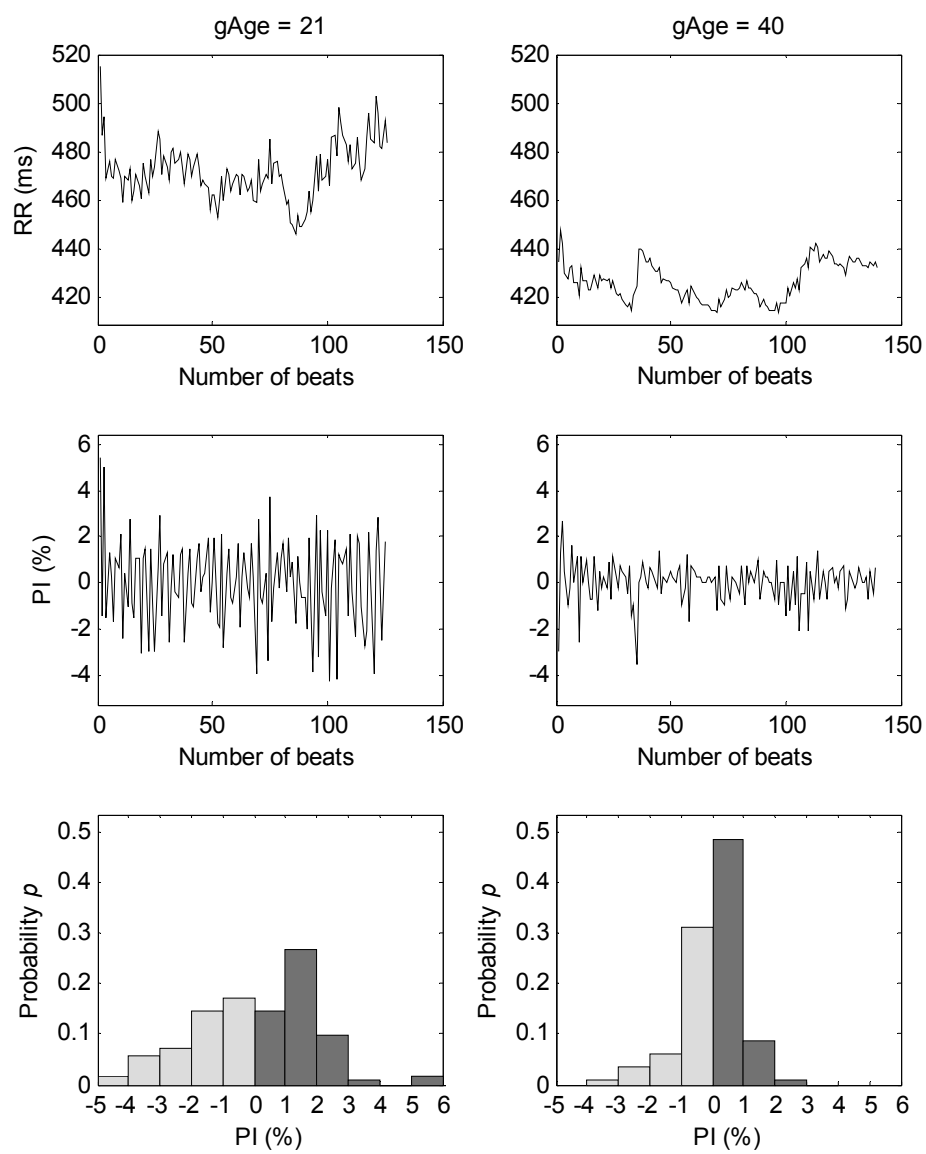

Figure 1. Typical heart period (RR intervals) time series (upper), PI time series at lag 1 (middle), and its probability distributions in histogram (bottom) selected in each group (G1 and G2). Abbreviations are defined in the text.
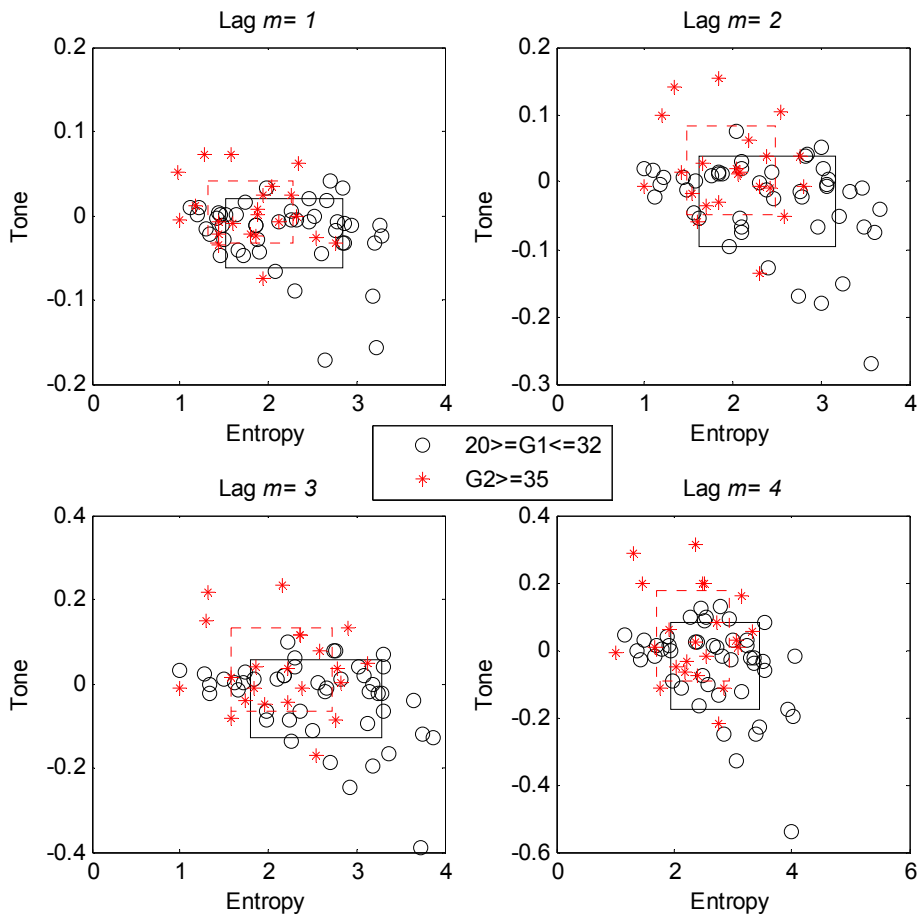

Figure 2. Evaluated tone and entropy in T-E space (lags 1-4) with averages by open rectangles (averages $\pm \mathrm{SE}$ ), and individuals, by symbols. 
Table 1. Mean $\pm \mathrm{SD}$ values of tone and entropy parameters at lags $\mathrm{m}=1-8 . p<0.05$ is significant. Tones are significantly different at all lags between early and late goups however, entropy is only different up to lag 4. NS means non significant.

\begin{tabular}{|c|c|c|c|c|}
\hline Parameter & Lag m & G1 (46) Mean \pm SD & G2 (22) Mean \pm SD & $\boldsymbol{p}$ \\
\hline \multirow{5}{*}{ Tone } & 1 & $-0.02 \pm 0.04$ & $0.00 \pm 0.04$ & 0.019 \\
& 2 & $-0.03 \pm 0.07$ & $0.02 \pm 0.07$ & 0.009 \\
& 3 & $-0.04 \pm 0.09$ & $0.03 \pm 0.10$ & 0.008 \\
& 5 & $-0.05 \pm 0.13$ & $0.04 \pm 0.14$ & 0.013 \\
& 6 & $-0.05 \pm 0.16$ & $0.05 \pm 0.17$ & 0.014 \\
& 7 & $-0.06 \pm 0.19$ & $0.06 \pm 0.20$ & 0.023 \\
& 8 & $-0.07 \pm 0.22$ & $0.07 \pm 0.23$ & 0.026 \\
& 1 & $2.18 \pm 0.66$ & $1.80 \pm 0.49$ & 0.019 \\
& 2 & $2.39 \pm 0.77$ & $1.98 \pm 0.51$ & 0.028 \\
& 3 & $2.54 \pm 0.74$ & $2.15 \pm 0.58$ & 0.034 \\
& 4 & $2.70 \pm 0.76$ & $2.33 \pm 0.62$ & 0.049 \\
& 5 & $2.84 \pm 0.78$ & $2.51 \pm 0.65$ & NS \\
& 5 & $2.96 \pm 0.78$ & $2.63 \pm 0.69$ & NS \\
& 6 & $3.05 \pm 0.78$ & $2.71 \pm 0.69$ & NS \\
& 7 & $3.14 \pm 0.78$ & $2.82 \pm 0.66$ & NS \\
\hline \multirow{5}{*}{ Entropy } & 8 & & &
\end{tabular}

Mean tone and entropy values for both the G1 and G2 group for all lags $(1 \leq \mathrm{m} \leq 8)$ are shown in Figure 3. Mean tone values are lower for all lags in the G1 group compared to the G2 group (upper panel). In addition mean tone values in the G1 group consistently decrease with increasing lag. On the other hand, mean entropy values are higher in the G1 than G2 group for all lags (Figure 3 lower panel). The mean entropy values in both groups are mostly increased with increasing lags (Table 1, Figure 3 ).

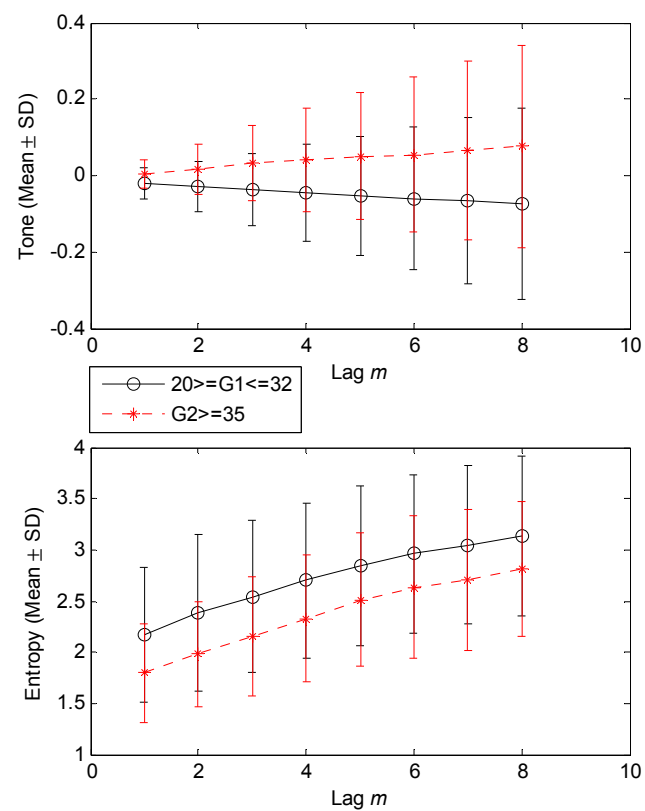

Figure 3. Tone-entropy response to changing lag $\mathrm{m}(1-8)$ in early foetuses (G1) and late foetuses (G2). Table 1 summarizes the values. 
The relationships of tone and entropy at multiple lags (1-4) with gestational ages are illustrated in Figures 4 and 5. Tone was increasing with gestational ages however entropy was decreasing with gestational ages. Significant $(p<0.05)$ correlations were only seen at lags 2 and 3 for tone values and 1 and 2 for entropy values. Table 3 shows that there are no significant differences in meanRR, $S D R R$ and $R M S S D$ values between G1 and G2 groups.
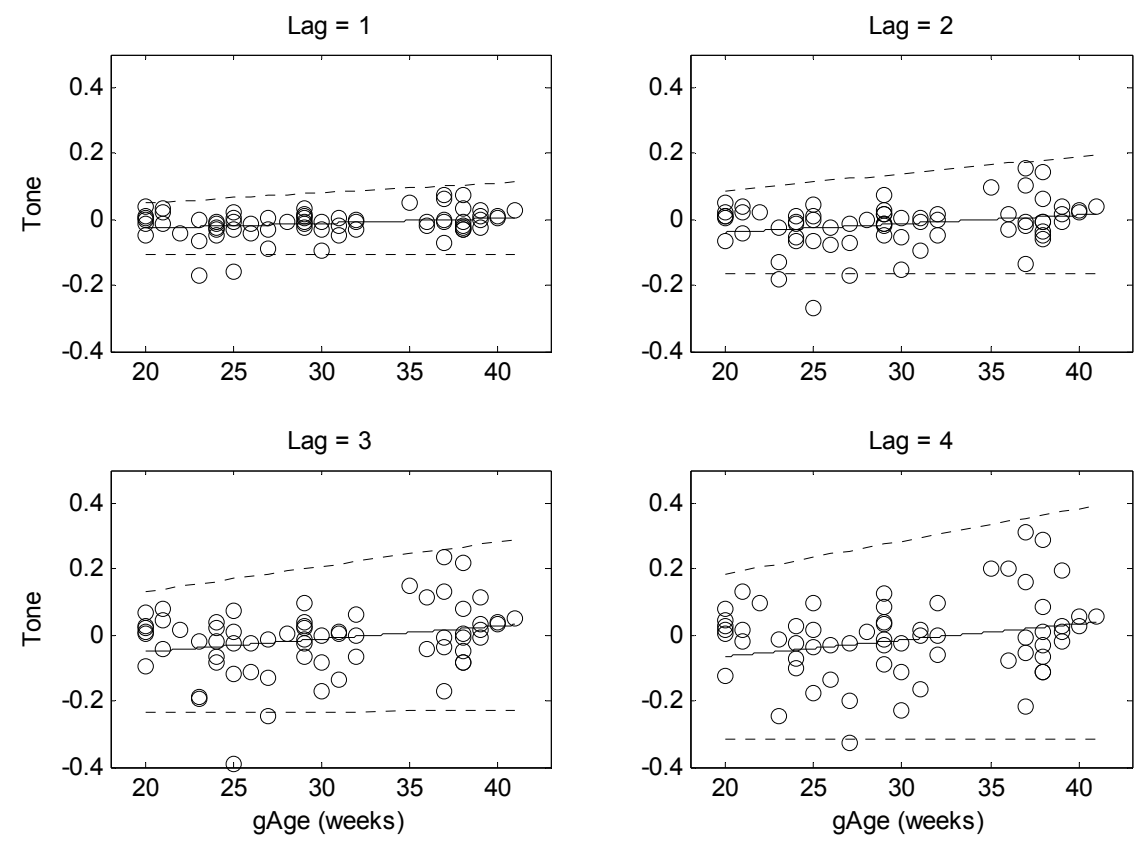

Figure 4. Correlation between tone and gestational age at lags $m=1-4$. Table 2 summarizes the values (correlation coefficients and significance). Solid lines represent the means and dotted lines represent the $95 \%$ confidence levels.
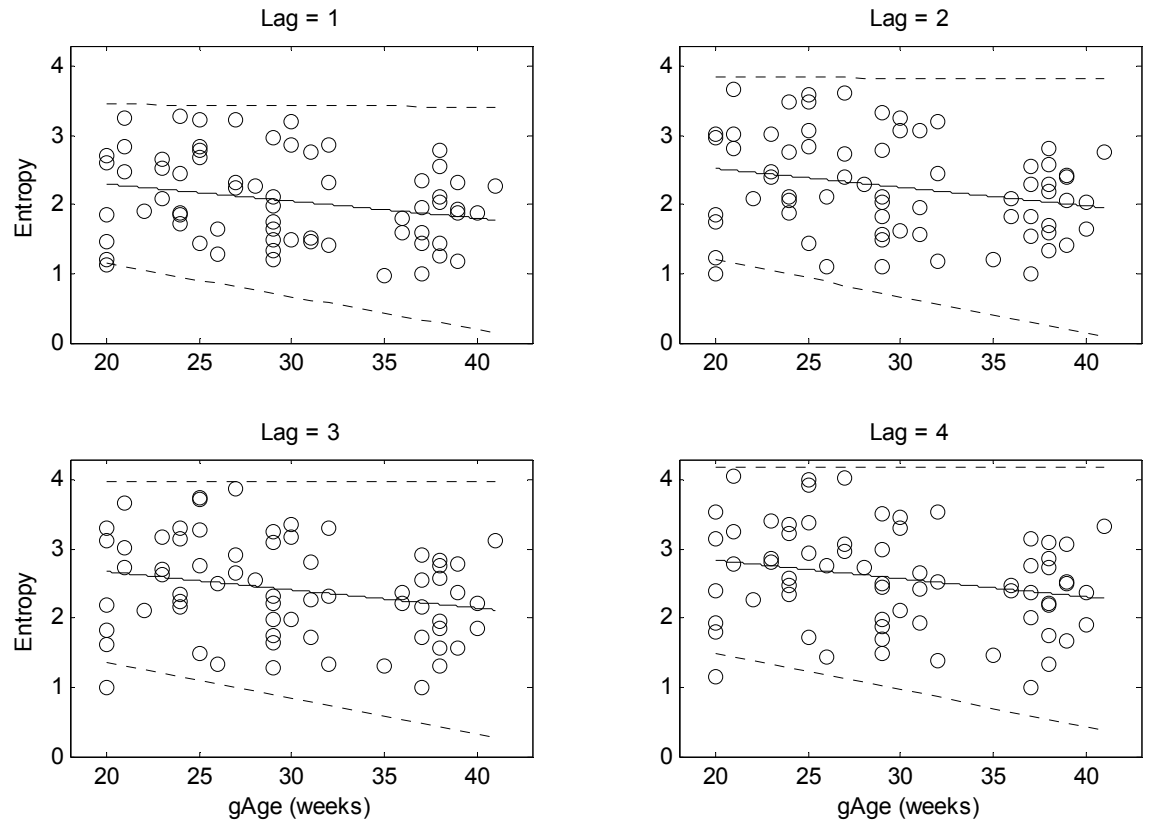

Figure 5. Correlation between entropy and gestational age at lags $m=1-4$. Table 2 summarizes the values (correlation coefficients and significance). Solid lines represent the means and dotted lines represent the $95 \%$ confidence levels. 
Table 2. Spearman correlation coefficient (R) with gestational ages and corresponding $p$ values for alllags. NS means non significant.

\begin{tabular}{|c|c|c|c|}
\hline Parameter & Lag $\boldsymbol{m}$ & $\mathbf{R}$ & $\boldsymbol{p}$ \\
\hline \multirow{5}{*}{ Tone } & 1 & 0.23 & 0.05 \\
& 2 & 0.25 & 0.04 \\
& 3 & 0.25 & 0.04 \\
& 4 & 0.24 & 0.05 \\
& 5 & 0.23 & 0.05 \\
& 6 & 0.21 & $\mathrm{NS}$ \\
& 7 & 0.21 & $\mathrm{NS}$ \\
& 8 & 0.20 & $\mathrm{NS}$ \\
\hline \multirow{5}{*}{ Entropy } & 1 & -0.26 & 0.03 \\
& 2 & -0.24 & 0.04 \\
& 3 & -0.24 & 0.05 \\
& 4 & -0.24 & 0.05 \\
& 5 & -0.20 & $\mathrm{NS}$ \\
& 6 & -0.20 & $\mathrm{NS}$ \\
& 7 & -0.20 & $\mathrm{NS}$ \\
& 8 & -0.20 & $\mathrm{NS}$ \\
\hline
\end{tabular}

Table 3. Mean \pm SD values of time domain parameters. $p<0.05$ is significant. None of them are significantly different.

\begin{tabular}{cccc}
\hline Parameter & G1 (46) Mean \pm SD & G2 (22) Mean \pm SD & $\boldsymbol{p}$ \\
\hline MeanRR $(\mathrm{ms})$ & $416.4 \pm 28.0$ & $409.3 \pm 29.8$ & 0.34 \\
SDRR $(\mathrm{ms})$ & $13.0 \pm 9.1$ & $9.9 \pm 5.7$ & 0.14 \\
RMSSD $(\mathrm{ms})$ & $6.6 \pm 4.1$ & $4.7 \pm 3.8$ & 0.09 \\
\hline
\end{tabular}

\section{Discussion}

Linear and nonlinear analysis of foetal heart rate variability have been applied for some time for the classification of abnormal and compromised foetuses [13,14]. These methods differ in terms of their appropriateness for analysis of the foetal HRV (fHRV) including the measurement of foetal heart rate techniques. This opened up the question of finding a method that allows measuring this multi-lag characteristic of beat to beat heart rate time series and its usefulness in clinical application.

The question this paper addressed was whether multi-lag T-E values of fHRV can be used to correctly identify a foetus as early (before 32 weeks) or late (after 35 weeks), which takes into consideration the influence an individual beat has on subsequent beats in the time series. The physiological interpretations of tone and entropy in various experimental settings were previously reported [5,15]. Lower tone values (negative) indicate that vagal activity predominates in the sympatho-vagal balance in a healthy population at rest $[5,16,17]$. Higher tone (nearing zero or positive) and lower entropy values indicate that parasympathetic efferent pathways are progressively withdrawing their activity [15]. The main novelty of this work is to look at the tone and entropy values of fHRV at multiple lags with progress of gestational ages (early and late fetuses). The major findings of the study are discussed in the following subsections. 


\subsection{Changes of T-E Values at Different Lags}

The mean tone value for the early foetus group decreased with increasing lags. This might indicate that the parasympathetic influence of the current beat increases with increasing lag (distant heart beats) in early foetuses. On the other hand, the mean tone value for the late foetus group increased with increasing lag which could mean that sympathetic influence of the current beat increases with increasing lag. Therefore, tone at different lags can be considered as a measurement of sympatho-vagal balance at multiple lags with the lower lags (approx. 1 to 5) predominantly indicating parasympathetic influence. The mean entropy values for both early and late foetuses increased with increasing lag. Tone values at lag 2 and 3 are more significant than that at lag 1. However, entropy values for late foetuses were significantly lower than those of early group up to lag 4.

T-E has two major advantages over the conventional methods in that it is robust against non-stationarities and not sensitive to differences in length of recordings. This latter point makes it especially useful for clinical investigation where short time recordings are more likely to be taken [15]. In our previous study [15], the physiological implication of a T-E trace on a curvi-linear relation with a series of autonomic perturbations experiments was described. The T-E analysis results quantified parasympathetic nervous system activity during these perturbations as part of the sympatho-vagal control mechanism of heart function.

\subsection{Gradual Increase in Sympathetic Activity with Foetal Cardiovascular Development}

A histologic study of the cardiac conduction system in human foetal hearts has found that the sinus node is completely developed by the 6th gestational week, the atrioventricular node by the 10th gestational week, and the entire functional conduction system by the 16th gestational week [18]. Clinical observation also suggests that foetal autonomic nerves can be analyzed starting in the 17th gestational week [1]. The previous study results [1] on low frequency (LF) power of foetal HRV (Figure 6) suggest that parasympathetic nerves, which develop rapidly in the 18th gestational week, are not involved in the increase in LF power. Development of the sympathetic nerves begins around the 20th gestational week, later than does that of the parasympathetic nerves, and is most rapid during the 26th to 30th gestational weeks, which coincide with the period of rapid increase in the LF power [1]. Another study [19] reported that LF/HF ratio was enhanced at 32 weeks or later, which was consistent with previous report [20] of a conversion point at 32 weeks of gestation. These neural control mechanisms will nearly reach the neonatal level in the 37th gestational week. Therefore, the maturity of the foetal autonomic nerve can be evaluated by calculating TE as pregnancy progresses. The maturity of foetal autonomic nervous system night also be related to the development of foetal behavioral patterns which are known to change with gestational ageing, particularly increasing frequency of movement is noticed in late foetuses. In human neonates, studies confirmed that during sustained stimulus presentation HRV was reduced and individual differences in baseline HRV were correlated with heart rate reactivity [21]. 


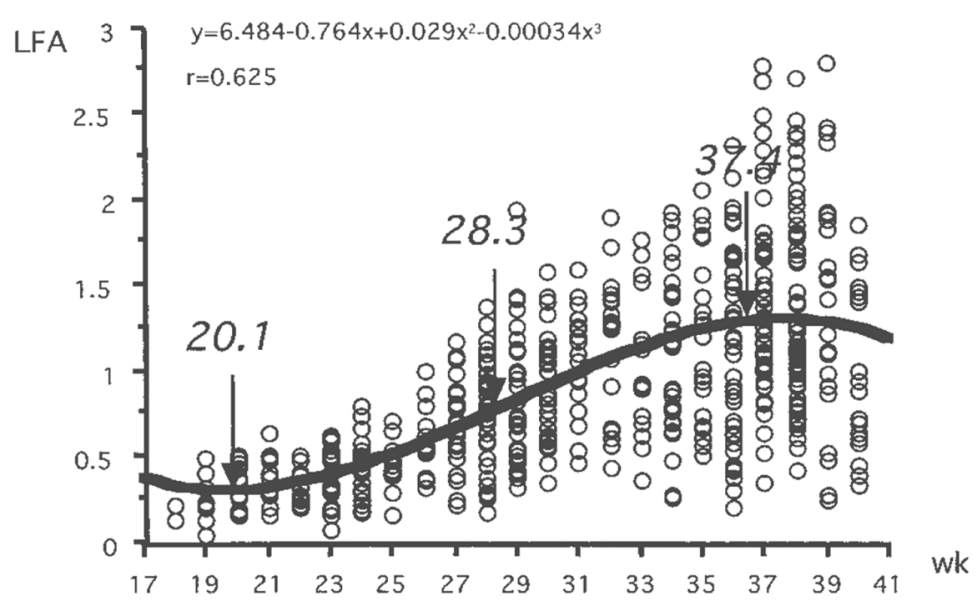

Figure 6. Alteration of low-frequency area (LFA) of the spectrum of heart beat fluctuation in normal foetuses during pregnancy [1].

We suggest that the present results of increasing tone of the late foetus group is an alternate expression of this increased sympathetic activity and decreasing entropy as a reduction of HRV because entropy represents the total acceleration-inhibition activity of the cardiac autonomic nervous system function and outcome. Also, it was verified that tone was increased when HRV is significantly depressed after blockade of parasympathetic efferent pathways and the resulting dominance of the sympathetic division [15].

\subsection{Alteration of Sympatho-Vagal Balance with the Gestational Ages}

The high entropy values, concomitant with lower (negative) values of tone, observed for early foetuses (Figure 4) could indicate that the development of two cardiac autonomic pathways are always active and that vagal activity predominates in the sympatho-vagal balance. In our previous study [15], it was shown that administration of scopolamine (vagal stimulation drug) increased the entropy values and reduced the tone values. Thus, our results may infer that foetal development causes an alteration in the T-E balance that correlates with the progressive innervations of sympathetic activity towards delivery. Low birth weight babies and premature birth are major problems in obstetrics community at present. We expect that the assessment of fetal development by T-E method will become a useful tool in clinical phase in near future. The limitations of this study are that: (i) there are no direct comparisons of T-E values with standard HRV indices including acceleration/deceleration capacity presented in the results, (ii) gender of fetuses were not considered.

\section{Conclusions}

Multi-lag T-E values of fHRV which take the influence an individual beat on subsequent beats in the time series into consideration, may be used to identify the progression of autonomic nervous system development in foetus as early (before 32 weeks) or late (after 35 weeks). Increasing tone of late foetus group is an alternate expression of this increased sympathetic activity and decreasing entropy as a reduction of HRV. Tone and entropy values of fHRV could be useful in clinical scenarios where short term recordings are frequently used for clinical diagnosis. 


\section{Acknowledgments}

This work was supported by an Australian Research Council Linkage under Grant LP100200184 between the University of Melbourne, Tohoku University and Atom Medical Corporation in Japan. A part of the work was carried out under the Coordination, Support and Training Program for Translational Research, Ministry of Education, Culture, Sports, Science and Technology in Japan.

\section{Author Contributions}

Ahsan Khandoker conceived, derived and implemented the tone entropy analysis, generated experimental results data and drafted the Manuscript. Chandan Karmakar generated the plots for multi-lag tone entropy analysis and drafted the Manuscript. Miyuki Endo, Sayaka Oshio and Yoshitaka Kimura carried out the fetal ECG experiments at Tohoku University Hospital. Yoshitaka Kimura and Marimuthu Palaniswami participated in the discussion and interpretation of the results. All authors have read and approved the final manuscript.

\section{Conflicts of Interest}

The authors declare no conflict of interest

\section{References}

1. Ohta, T.; Okamura, K.; Kimura, Y.; Suzuki, T.; Watanabe, T.; Yasui, T.; Yaegashi, N.; Yajima, A. Alteration in the low-frequency domain in power spectral analysis of fetal heart beat fluctuations. Fetal Diagn. Ther. 1999, 14, 92-97.

2. Renou, P.; Newman, W.; Wood, C. Autonomic control of fetal heart rate. Am. J. Obstet. Gynecol. 1969, 105, 949-953.

3. Gagnon, R.; Campbell, K.; Hunse, C.; Patrick, J. Patterns of human-fetal heart-rate accelerations from 26 weeks to term. Am. J. Obstet. Gynecol. 1987, 157, 743-748.

4. Druzin, M.L. Antepartum fetal heart-rate monitoring. State of the art. Clin. Perinatol. 1989, 16, 627-642.

5. Oida, E.; Moritani, T.; Yamori, Y. Tone-entropy analysis on cardiac recovery after dynamic exercise. J. Appl. Physiol. 1997, 82, 1794-1801.

6. Oida, E.; Kannagi, T.; Moritani, T.; Yamori, Y. Aging alteration of cardiac vagosympathetic balance assessed through the tone-entropy analysis. J. Gerontol. A 1999, 54, M219-M224.

7. Karmakar, C.K.; Khandoker, A.H.; Jelinek, H.F.; Palaniswami, M. Risk stratification of cardiac autonomic neuropathy based on multi-lag tone-entropy. Med. Biol. Eng. Comput. 2013, 51, 537-546.

8. Lerma, C.; Infante, O.; Perez-Grovas, H.; Jose, M.V. Poincare plot indexes of heart rate variability capture dynamic adaptations after haemodialysis in chronic renal failure patients. Clin. Physiol. Funct. Imaging 2003, 23, 72-80.

9. Sato, M.; Kimura, Y.; Chida, S.; Ito, T.; Katayama, N.; Okamura, K.; Nakao, M. A novel extraction method of fetal electrocardiogram from the composite abdominal signal. IEEE Trans. Biomed. Eng. 2007, 54, 49-58. 
10. Pan, J.; Tompkins, W.J. A real-time QRS detection algorithm. IEEE Trans. Biomed. Eng. 1985, 32, 230-236.

11. Amano, M.; Oida, E.; Moritani, T. Age-associated alteration of sympatho-vagal balance in a female population assessed through the tone-entropy analysis. Eur. J. Appl. Physiol. 2005, 94, 602-610.

12. Shannon, C.E. A mathematical theory of communication. Bell Syst. Tech. J. 1948, 27, 379-423.

13. Gierałtowski, J.; Hoyer, D.; Tetschke, F.; Nowack, S.; Schneider, U.; Żebrowski, J. Development of multiscale complexity and multifractality of fetal heart rate variability. Auton. Neurosci. 2013, 178, 29-36.

14. Van Leeuwen, P.; Cysarz, D.; Edelhäuser, F.; Grönemeyer, D. Heart rate variability in the individual fetus. Auton. Neurosci. 2013, 178, 24-28.

15. Khandoker, A.H.; Jelinek, H.F.; Moritani, T.; Palaniswami, M. Association of cardiac autonomic neuropathy with alteration of sympatho-vagal balance through heart rate variability analysis. Med. Eng. Phys. 2010, 32, 161-167.

16. Bootsma, M.; Swenne, C.A.; Vanbolhuis, H.H.; Chang, P.C.; Cats, V.M.; Bruschke, A.V.G. Heart-rate and heart-rate-variability as indexes of sympathovagal balance. Am. J. Physiol. 1994, 266, H1565-H1571.

17. Tulppo, M.P.; Hughson, R.L.; Makikallio, T.H.; Airaksinen, K.E.J.; Seppanen, T.; Huikuri, H.V. Effects of exercise and passive head-up tilt on fractal and complexity properties of heart rate dynamics. Am. J. Physiol. Heart Circ. Physiol. 2001, 280, H1081-H1087.

18. James, T.N. Cardiac conduction system: Fetal and postnatal development. Am. J. Cardiol. 1970, 25, 213-226.

19. Assali, N.S.; Brinkman, C.R.; Woods, J.R.; Dandavino, A.; Nuwayhid, B. Development of neurohumoral control of fetal, neonatal, and adult cardiovascular functions. Am. J. Obstet. Gynecol. 1977, 129, 748-759.

20. Hayashi, R.; Nakai, K.; Fukushima, A.; Itoh, M.; Sugiyama, T. Development and significance of a fetal electrocardiogram recorded by signal-averaged high-amplification electrocardiography. Int. Heart J. 2009, 50, 161-171.

21. Porges, S.W. The polyvagal theory: Phylogenetic substrates of a social nervous system. Int. J. Psychophysiol. 2001, 42, 123-146.

(C) 2015 by the authors; licensee MDPI, Basel, Switzerland. This article is an open access article distributed under the terms and conditions of the Creative Commons Attribution license (http://creativecommons.org/licenses/by/4.0/). 